\title{
Glucocorticoid-Induced Cardioprotection: A Novel Role for Autophagy?
}

\author{
Anna-Mart Engelbrecht and Benjamin Loos
}

Additional information is available at the end of the chapter

http://dx.doi.org/10.5772/52406

\section{Introduction}

Glucocorticoids (GC) are commonly used as anti-inflammatory and immunosuppressive therapy by approximately $1 \%$ of the total adult population. Glucocorticoid therapy has also been used in non-autoimmune and non-inflammatory conditions such as acute myocardial infarction, angina, endocarditis as well as in invasive cardiology, coronary interventions and cardiopulmonary- bypass surgery. Despite ample evidence for GC's role as a natural, physiologic regulator of the immune system, little is known about the molecular events induced by GCs during a stress response. Autophagy is a survival mechanism which is upregulated in response to stress in the cell. It has been described as the cell's major adaptive strategy in response to a multitude of extracellular stresses, such as nutrient deprivation, mitochondrial damage, endoplasmic reticulum stress or infection. Conserved in all eukaryotes, it is mediated by a unique organelle, the autophagosome, which, under inclusion of cytoplasmic cargo, fuses with lysosomes in order to yield recyclable nutrient metabolites. Basal autophagic activity plays a vital role in maintaining homeostasis during cellular stress. Its malfunction has been implicated with human pathologies such as heart disease, neurological storage disease and cancer.

It is known that GC-triggered autophagy plays a role in cell death during development. However, recent landmark studies also indicate that autophagy operates as a hub, integrating cellular stress, metabolism and glucocorticoid mediated anti-inflammatory action. Importantly, recent lines of evidence suggest that glucocorticoids impact on key signalling components, which control the activity of the autophagic machinery.

In this review we will focus on the connections between these key signaling components and autophagy, describing their central roles as modulators of GC-induced protection during a cellular stress response. 


\section{Glucocorticoid generation and metabolism}

The understanding of the physiological regulation of glucocorticoid activity has considerably improved over the last few decades. The generation of glucocorticoids from cholesterol, which occurs in the zonae fasiculata and reticularis of the adrenal cortex, is tightly regulated by the hypothalamic-pituitary-adrenal (HPA) axis where glucocorticoids regulate its own generation through negative feedback inhibition. Glucocorticoids are produced de novo under this control and are released into the blood as required, with a definite circadian rhythm producing peak concentrations in the early morning [1]. When secreted into the blood, most (90-95\%) of glucocorticoids are sequestered to corticosteroidbinding globulin and albumin with the unbound fraction available to interact with their receptors [2]. Metabolic inactivation of glucocorticoids occurs predominantly in the liver, but also in the kidney with inactive metabolites excreted in the urine.

\section{Molecular actions of glucocorticoids: Genomic and non-genomic pathways}

The classical mode of glucocorticoid-induced gene expression, i.e. the genomic effect involves ligand-dependent activation and release from chaperone proteins (heat shock protein-90 and others), translocation of the receptor-complex to the nucleus where binding of the glucocorticoid receptor to glucocorticoid response element (GRE) in the promoter of the target genes will lead to transcriptional activation of the genes within hours [3, 4]. Activation of glucocorticoid-responsive genes occurs via interaction between the DNAbound GR and transcriptional co-activator molecules such as CREB-binding protein, which have intrinsic histone acetyltransferase activity and cause acetylation of core histones. This tags histones to recruit chromatin remodeling engines and subsequent association of RNA polymerase II resulting in gene activation [5]. Increasing evidence suggests that glucocorticoids can also cause rapid activation of signaling molecules prior to altering gene expression. These so called non-genomic effects occur within minutes of glucocorticoid exposure and are not affected by inhibiting RNA transcription [5, 6].

Metabolic effects of glucocorticoids represent most of the adverse effects of glucocorticoid therapy and are mainly ascribed to the transcriptional activity of the glucocorticoid receptor, whereas the therapeutically beneficial anti-inflammatory actions are thought to be predominantly caused by the mechanism of transrepression where the activated GR can selectively repress the transcription of specific inflammatory genes without binding to DNA itself but by a number of pleiotropic actions at the promoters of inflammatory genes. Inflammatory genes are regulated by the actions of proinflammatory transcription factors such as nuclear factor- $\mathrm{\kappa B}(\mathrm{NF}-\kappa \mathrm{B})$, activator protein -1 (AP-1), and signal transducer and transcription proteins. Activated GR binds to these transcription factors, either directly or indirectly, and recruits co-repressor proteins that blunt the ability of these transcription factors to switch on inflammatory genes [7]. Furthermore, many pro-inflammatory genes are repressed by GC at post-transcriptional level via mRNA destabilization or inhibition of 
translation, however, this phenomenon cannot be accounted for by transrepression, therefore suggest the existence of an additional anti-inflammatory mechanism of GCs [8].

\section{Ischemia/reperfusion-induced stress in the heart}

Glucocorticoids play a key role in the response to stress in the heart where it can influence the regulation of blood pressure, inflammation, immune function and cellular energy metabolism [9-10]. These acute effects contribute to an adaptive response in the short term. Although the cardioprotective effects of glucocorticoids in the acute setting of ischemia/reperfusion have been experimentally demonstrated in animals [11-13] and humans [14], the molecular mechanisms still need to be fully elucidated.

Ischemia can be defined as an imbalance between the amount of oxygen, glucose and other substrates needed by the heart $[15,16]$. This leads to anaerobic metabolism and reduced contractile function. A biochemical imbalance occurs as the maintenance of the metabolism cannot be kept at a steady state due to inadequate coronary flow. A reduction in metabolite clearance also occurs during ischemia and intracellular $\mathrm{pH}$ levels drop as the acid byproducts of glycolysis accumulate. The severity of ischemic injury depends on the duration of ischemia and subsequent reperfusion $[15,16]$. If, ischemia is maintained, reversible injury gradually transitions to irreversible injury and a myocardial infarct develops. Reperfusion with its reinforced oxygen and substrate availability is thus a prerequisite for myocardial salvage [16]. However, reperfusion after an ischemic period causes generation of free radicals and is associated with detrimental changes such as enzyme release, arrhythmias and intramyocardial haemorrhage which are known as reperfusion injuries [17]. Cardiomyocytes are highly dependent on a continuous supply of oxygen. During ischemia, cardiomyocyte capacity to generate sufficient ATP and creatine phosphate becomes depleted and multiple adaptive processes occur in response to these hypoxic environments created during ischemia. To reduce oxygen consumption, oxidative phosphorylation is limited and glycolysis is stimulated. This aids in ATP production, even under low oxygen supply [18]. Prolonged ischemia leads to cardiac failure which is characterized by the progressive death of myocytes [19]. Three major morphologies of cell death have been described, viz. apoptosis (type I), cell death associated with autophagy (type II) and necrosis (type III).

\section{The basic mechanisms of Autophagy}

Autophagy, from greek self eating, is a conserved degradation and recycling system for longlived proteins and other sub-cellular constituents. This degradation system is inherent to all eukaryotes and is mediated by a unique organelle, the autophagosome, which, under inclusion of cytoplasmic cargo, fuses with lysosomes in order to yield recyclable nutrient metabolites. Although already described in 1966 by de Duve and Wattiaux [20], Autophagy has received significant renewed attention in the last years. This new interest is primarily based on the recently gained understanding of the molecular components of the autophagic machinery. Genetic analyses in yeast identified more than 30 autophagy-related genes (ATG), and their corresponding proteins (Atg) participating in the autophagic pathway [21]. 
Multiple mechanisms exist for the mode of delivery of cytoplasmic material to the lysosome, giving rise to different types of autophagy. While microphagy is characterized by cytoplasm engulfment directly at the lysosomal surface by invagination of its membrane, macroautophagy involves the synthesis of double-membrane vesicles, which sequester portions of the cytoplasm [22]. Chaperone mediated autophagy (CMA) on the other hand involves selective motif tagged protein translocation directly through the lysosomal membrane [23]. However, shared by all three mechanisms is the final step of lysosomal cargo degradation by hydrolases, allowing the recycling of degraded material. Here we will focus on macroautophagy (herein referred to as autophagy), as it is the primary mechanism for cytoplasm-to-lysosome delivery.

The autophagic process can be divided into distinct steps, which include the induction, cargo packaging, vesicle nucleation, vesicle expansion and protein retrieval, docking and fusion and finally vesicle degradation [21]. In brief, the first event is the formation of the isolation membrane of the autophagosome. The Atg1 kinase complex governs these early steps in autophagosome formation. Central to this regulation is the nutrient sensor kinase mTOR (TORC). When mTOR is suppressed due to nutrient starvation, Atg1 kinase activity is triggered and affinity for Atg13 increases [24], which leads to the recruiting of other Atg proteins to initiate autophagosome formation. Cellular sources for this autophagosome formation step have been shown to be Golgi, ER, mitochondria and the plasma membrane [25]. During this process, two strongly interdependent conjugation systems are coordinating the events leading to the formation, elongation and sealing of the isolation membrane [26]. In the first conjugation system, Atg proteins 5, 7, 10 and 12 undergo a multimerization step with Atg16, leading to the formation of an Atg16 homotetramer, which assembles with four Atg12-Atg5 conjugates [27]. In the second conjugation system, the protein Atg8 is Atg4 dependently conjugated with phosphatidylethanolamine (PE) [28]. Reactive oxygen species play a role in controlling this step, as Atg4 oxidization enables autophagosome formation to proceed [29]. Next, two kinase complexes, PI3 kinase and Atg1, participate in the late stages of autophagosome formation. Atg6 (the mammalian orthologue, beclin-1) belongs to the PI3 kinase (PI3-K) class III complex. When Atg1 interacts with Atg13, progression towards a complete autophagosome takes place [27]. Cytoplasmic cargo is now confined. Docking and fusion with a lysosome will allow acidification of the autophagosome lumen, leading to the complete and rapid degradation of cargo into constituent components that are released into cytoplasmic space via permeases (Figure 1).

\section{Autophagy as a protective response during stress in the heart}

The terminally differentiated nature of cardiomyocytes demands a strong molecular reliance upon a functional autophagic degradation system. In cardiomyocytes autophagy has been described already in the late 1970's where it was emphasized as an important repair mechanism of sublethal injury [30]. Sybers and coworkers demonstrated the occurrence of myocyte autophagy in a fetal mouse heart that was kept for $1 \mathrm{~h}$ in organ culture. In addition they observed that Autophagy was accelerated by oxygen and glucose deprivation, but the hearts function could be restored following resupply of glucose and oxygen. However, 
when the period of injury lasted for longer than four hours, necrotic cell death was induced [30]. To date, many models have produced clear evidence that upregulation of autophagy promotes cell survival under conditions of metabolic perturbations and energy deprivation [31-33]. In the ischaemic myocardium, autophagy is upregulated rapidly following 20 minutes of coronary artery occlusion, leading to an increased number in autophagosomes [34]. In isolated cardiomyocytes exposed to anoxia-reoxygenation it was shown that inhibition of autophagy leads to an increase in necrotic cell death, which was further increased by additional inhibition of apoptosis [35]. However, enhancing autophagic flux, as indicated by an increased rate of autophagosomal clearance, protects cardiac myocytes against ischaemic injury by reducing apoptosis [31]. Moreover, the homeostatic role of functional basal autophagic activity in the myocardium has been demonstrated by cardiac specific disruption of Atg5, manifesting in impaired contractility, hypertrophy, dilation and sarcomeric disarray [36-37].

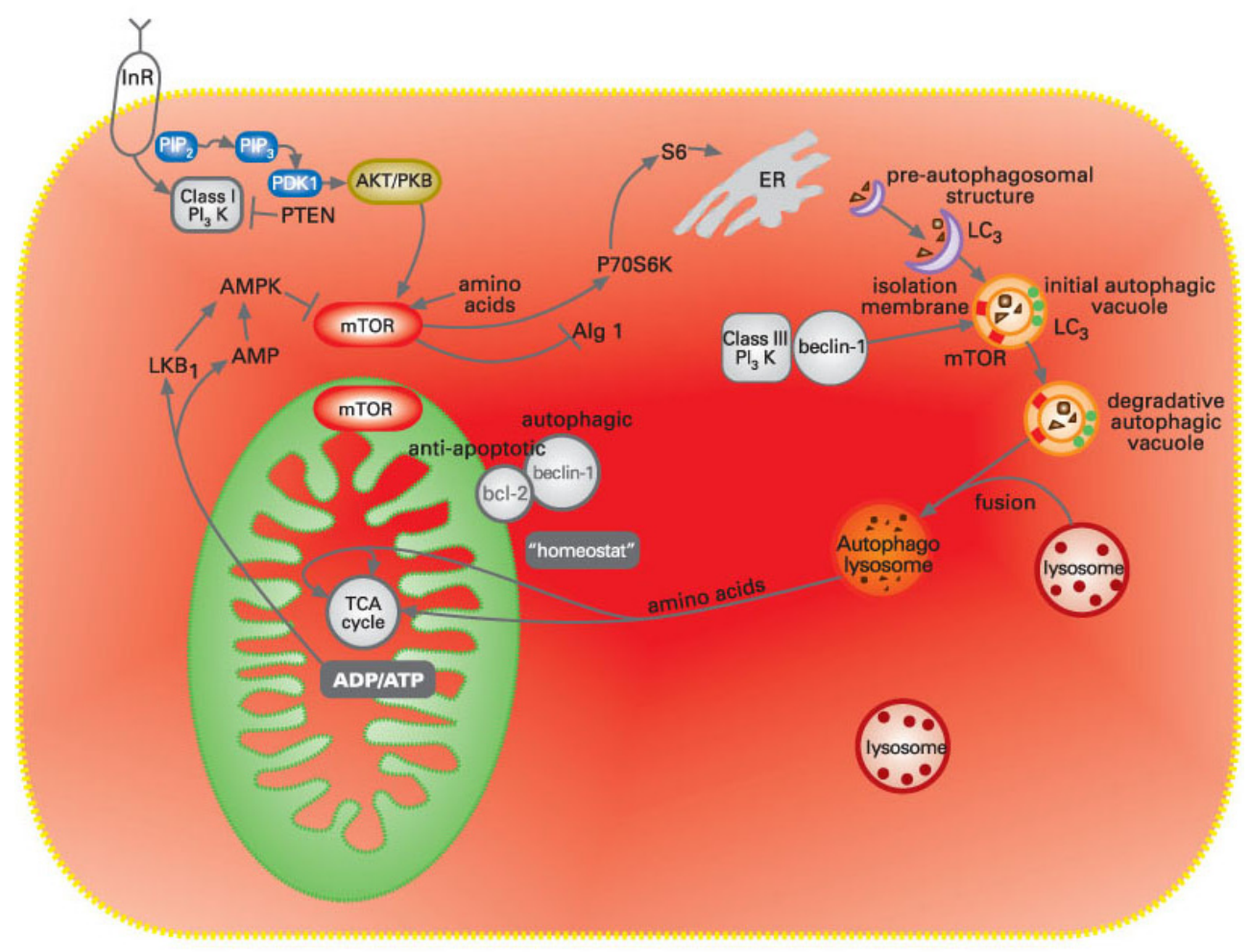

Figure 1. Schematic representation of the autophagic process, indicating the signalling network from induction to permease efflux with amino acid release, providing substrates for the TCA cycle. 


\section{Detrimental effects of Autophagy in the heart}

Literature suggests that autophagy is uniquely controlled during ischemia and reperfusion. A large body of evidence indicates that upregulation of autophagy particularly during the reperfusion phase is detrimental and exacerbates myocyte death. Energy sensing mediated by the $5^{\prime}$-AMP-activated protein kinase (AMPK) appears to be central to this control mechanism. In glucose-deprived cardiac myocytes, autophagy resulting from ischemia has been shown to be accompanied by AMPK activation, and was inhibited by dominant negative AMPK, suggesting an AMPK dependent mechanism [34]. AMPK is rapidly activated during myocardial ischemia, and leads to an increase in glucose uptake and oxidation as well as fatty acid oxidation [38]. Autophagy is enhanced after reperfusion, which is accompanied by an inactivation of AMPK and an increase in beclin-1 [34]. As AMPK switches off ATP-dependent processes, [39] its inactivation at reperfusion may contribute to an unfavorable metabolic environment. Moreover, data indicate that energy sensing mediated by AMPK is also differentially controlled depending on the severity of the ischaemic insult [40]. These reports are strengthened by recent data derived from cultured myoblasts, where a differential induction of cell death was observed, which was dependent on the severity and duration of the ischaemic insult [33]. Only mild ischaemic injury induced autophagy and apoptosis, while severe injury led to primarily necrotic cell death.

\section{Autophagy and myocardial metabolism}

The total cellular ATP amount in the cardiac myocyte is consumed in less than one minute [41] indicating the very high metabolic demand of the myocardium, and at the same time highlighting the existence of an extremely efficient system of energy conversion. In ischaemic conditions, energy metabolism is disrupted to a level where energy production cannot meet the myocardial energy demand. However, there is a clear role for autophagy in ischemia to influence the cell's energy profile, indicative to maintain metabolic supplydemand homeostasis [33]. ATP levels decrease rapidly with ischemia and recover rapidly after reperfusion [42]. These dynamics of ATP depletion become highly relevant when considering the molecular overlap between autophagy, apoptosis and necrosis [32; 43-44, Figure 2]. It has been demonstrated that an ATP depletion of $>50 \%$ is needed in order to change the mode of cell death from apoptotic to necrotic [45]. Vice versa, a progressive replacement of necrosis with apoptosis has been described, when intracellular ATP becomes available again [46]. Recent evidence strongly indicates the previously underestimated metabolic role of autophagy in generating metabolite substrates by shifting the cellular energetic balance [33; 47-49], suggesting that intracellular ATP availability may be controlled to a significant degree by the autophagic flux (Figure 2). These data strongly suggest that not only the magnitude of autophagic activity but also the cell's metabolic profile and microenvironment are crucial in controlling a favorable cellular response other than necrosis, and delaying apoptosis [32] (Figure 3). 


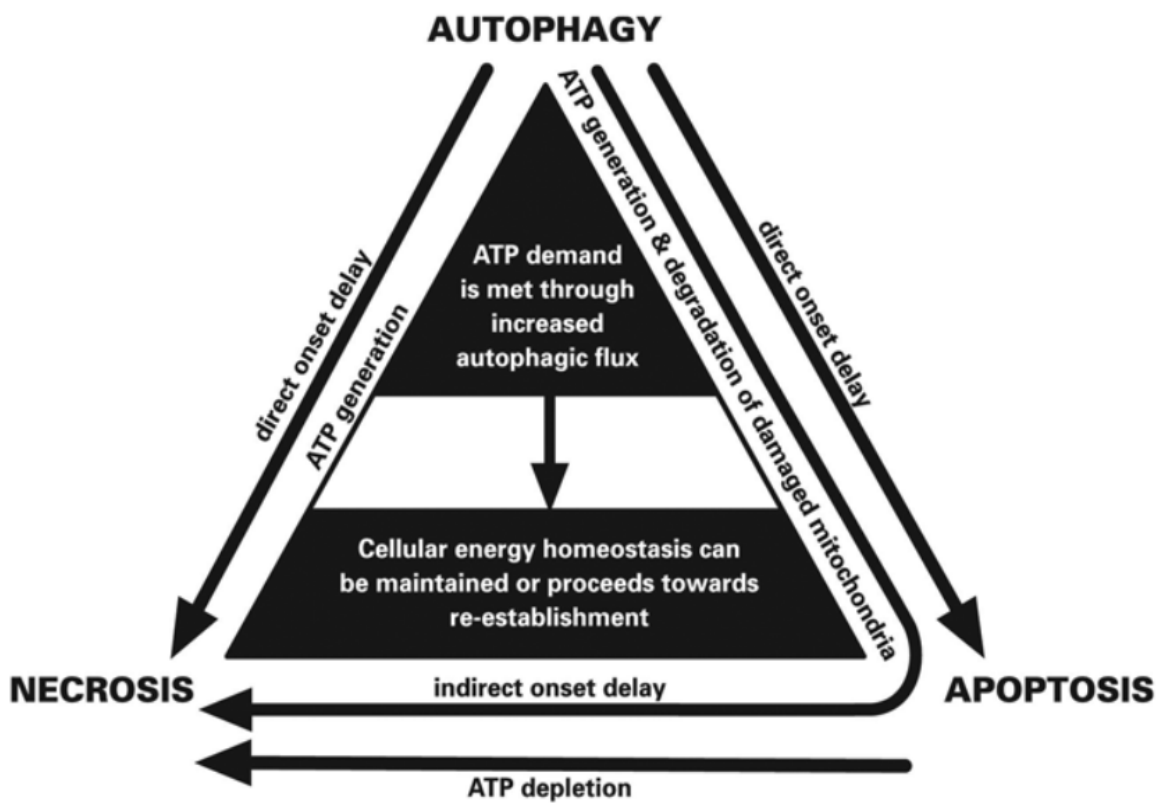

Figure 2. Recent evidence strongly indicates the previously underestimated metabolic role of autophagy in generating metabolite substrates and ATP by shifting the cellular energetic balance, with a direct and indirect effect on apoptosis and necrosis.

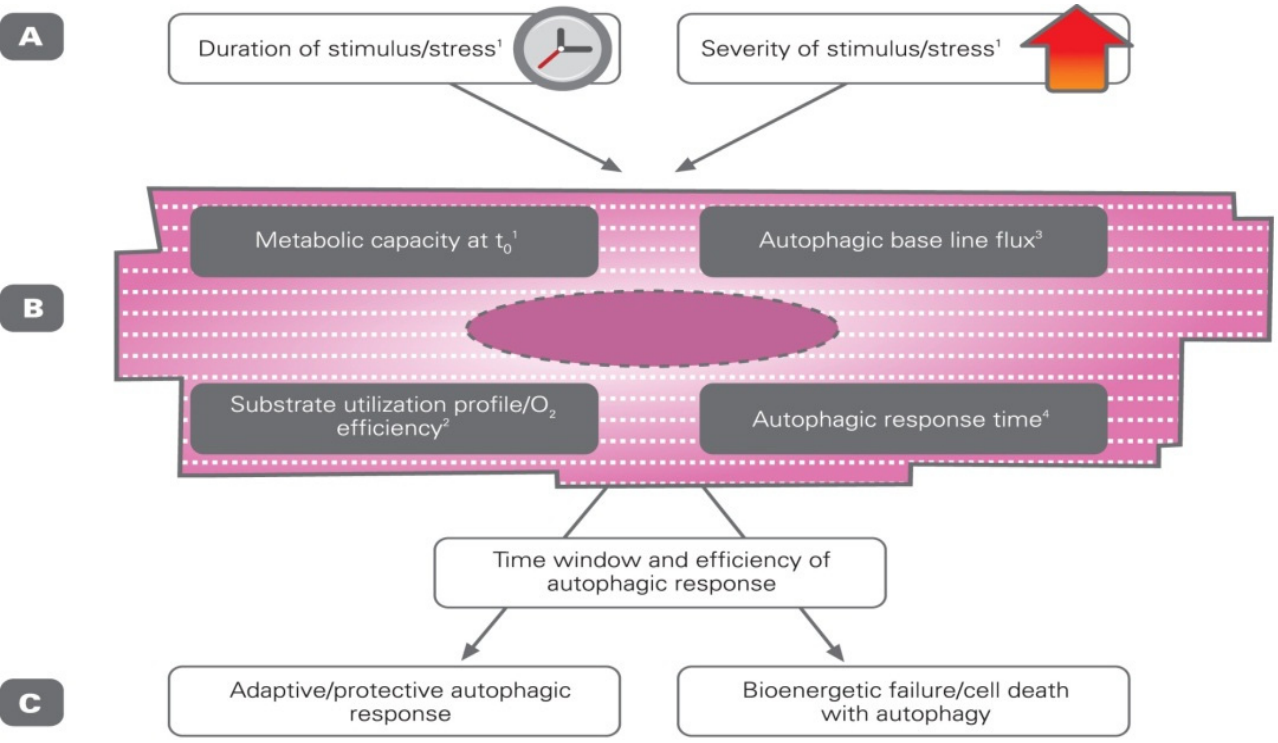

Figure 3. Whether autophagy may manifest in cytoprotection or type II programmed cell death is dependent on the severity and duration of the ischaemic insult, as well as autophagic flux- and metabolic parameters. 
The above results also stress the significance of the severity and duration of the ischaemic event, to allow a sufficient induction of the autophagic machinery to take place. In fact, a direct relationship between autophagic flux and myocardial function has recently been proposed [50], indicating the strong need to measure autophagic activity accurately. Further characterization of the autophagic flux in clear context with myocardial injury will help to answer questions when autophagy functions as a primarily destructive pathway, manifesting in type II programmed cell death or when autophagy functions in a cytoprotective manner.

\section{Autophagy and glucocorticoids-relationship and metabolic response}

The multifaceted relationship between autophagy and glucocorticoids is already indicated in embryogenesis. Recent reports not only demonstrate an important metabolic role for autophagy during embryogenesis and postnatal development [51,52], but also indicate a likely molecular link between autophagic programmed cell death and steroids during development [53]. Embryos from the atg5 ${ }^{--}$knockout mouse model die perinatally due to energy depletion, leading to a reduced plasma- and tissue amino acid concentration [54]. Moreover, increased apoptosis is displayed in various embryonic tissues derived from such embryos, supporting a role for autophagy in the removal of apoptotic bodies or in delaying the onset of apoptotic cell death [54]. Targeted disruption of beclin 1 in mice also leads to early death in embryogenesis [55]. Many examples of autophagy as a mode of programmed cell death during embryogenesis exist, suggesting that an important role for autophagic cell death in development. It has been shown that autophagic cell death requires the genes ATG7 as well as beclin 1 and can be induced by caspase- 8 inhibition [56]. In addition, embryonic fibroblasts from Bax/Bak double knockout mice undergo autophagic cell death, which can be suppressed by inhibitors of autophagy and which is dependent on ATG5. These data suggest a role for Bcl-2 family proteins controlling also non-apoptotic cell death in addition to regulating apoptosis [57]. Especially in lower eukaryotes, the rise in steroid titers can elicit a transcription regulatory hierarchy that results in synchronous autophagic cell death [53]. Such steroid triggered programmed autophagic cell death has been observed in larval salivary gland cells [53] as well as motorneurons [58]. These findings suggest that steroids can play a governing role in very specific scenarios, controlling autophagic activity and the duration of increased flux, which in turn can control the synchronous induction of cell death. It is however not known, whether a robust increase in cortisol release in humans following psychological stress, trauma, sepsis or starvation can elicit similar effects on autophagic activity during embryogenesis. Such studies deserve a great deal of attention.

Although limited data are available, similarities exist between the role of the autophagic pathway as a response mechanism to metabolic perturbations and glucocorticoids in the regulation of metabolic responses. Chronic excessive activation of glucocorticoid receptors leads to major cellular metabolic rearrangements such as insulin resistance, glucose intolerance and dyslipidaemia. Obesity and metabolic syndrome, which are characterized by a nutrient overload, have been associated with a hyperactivation of tissue mTOR, 
indicating a blunted autophagic response [59]. The systemic glucocorticoid excess is associated with an increase in cardiovascular risk factors [60]. One of the major causes of impact on these risk factors is thought to be the glucocorticoid mediated intravascular volume overload [60]. As the access of glucocorticoids to their receptors is controlled by the isozymes of 11- $\beta$-hydroxysteroid dehydrogenase in a tissue specific manner, makes manipulation of this pathway an attractive therapeutic target. By selective isozyme inhibitors, the glucocorticoid activity can be modulated locally, keeping systemic glucocorticoid concentrations within homeostatic range.

Also in the acute setting a relationship exists between glucocorticoid availability and autophagy induction. In the treatment of acute lymphoblastic leukemia, glucocorticoids are used as crucial therapeutic agent, due to their effect on inducing G1 phase cell cycle arrest and apoptosis. Recently it was shown that dexamethasone treatment induces cell death and involves the induction of autophagy before the onset of apoptosis [61]. Moreover, another level of interaction has been demonstrated as the role of autophagy in innate immunity has recently become clear. Both the ATG16L1 risk allele as well as ATG5 are selectively important for the function of the Paneth cell, a specialized epithelial cell in the small intestine [62]. Through genome-wide association screenings it was shown that the autophagic pathway plays a fundamental role in the predisposition to the inflammatory bowel condition Chron's disease [63]. Taken together, these data indicate the dynamic relationship between glucocorticoid-induced metabolic perturbations, autophagy induction, inflammation and cell death susceptibility. Further investigations are likely to provide new insights into this complex relationship to treat cardiovascular disease more effectively by exploiting the modulation of the autophagic machinery in context with controlling local glucocorticoid activity.

\section{The role of the mitogen-activated protein kinases (MAPKs) during ischemia/reperfusion-induced stress in the heart}

Great efforts have been made to disentangle the intricate relationship between signalling pathways and the stress response of the heart during ischemia/reperfusion-induced injury. Analysis is complicated due to the fact that several pathways can be activated simultaneously with differential effects. It has become however evident that the MAPKs are major mediators of I/R-induced injury. Recent data pin point the MAPK's as one of the crossroads between autophagy and glucocorticoid signalling events.

Three major classes of MAPKs (Figure 4), which include the extracellular signal regulated protein kinase (ERK)/p42/44, c-Jun NH2-terminal protein kinase (JNK)/stress activated protein kinase (SAPK) and p38 MAPK families have been identified [64,65]. The ERK pathway has been depicted as a pro-survival pathway and is activated by a variety of mitogens and phorbol esters [66,67]. The JNK and p38 MAPK pathways are regarded as proapoptotic pathways and are mainly activated by environmental stress and inflammatory cytokines $[67,68]$. 


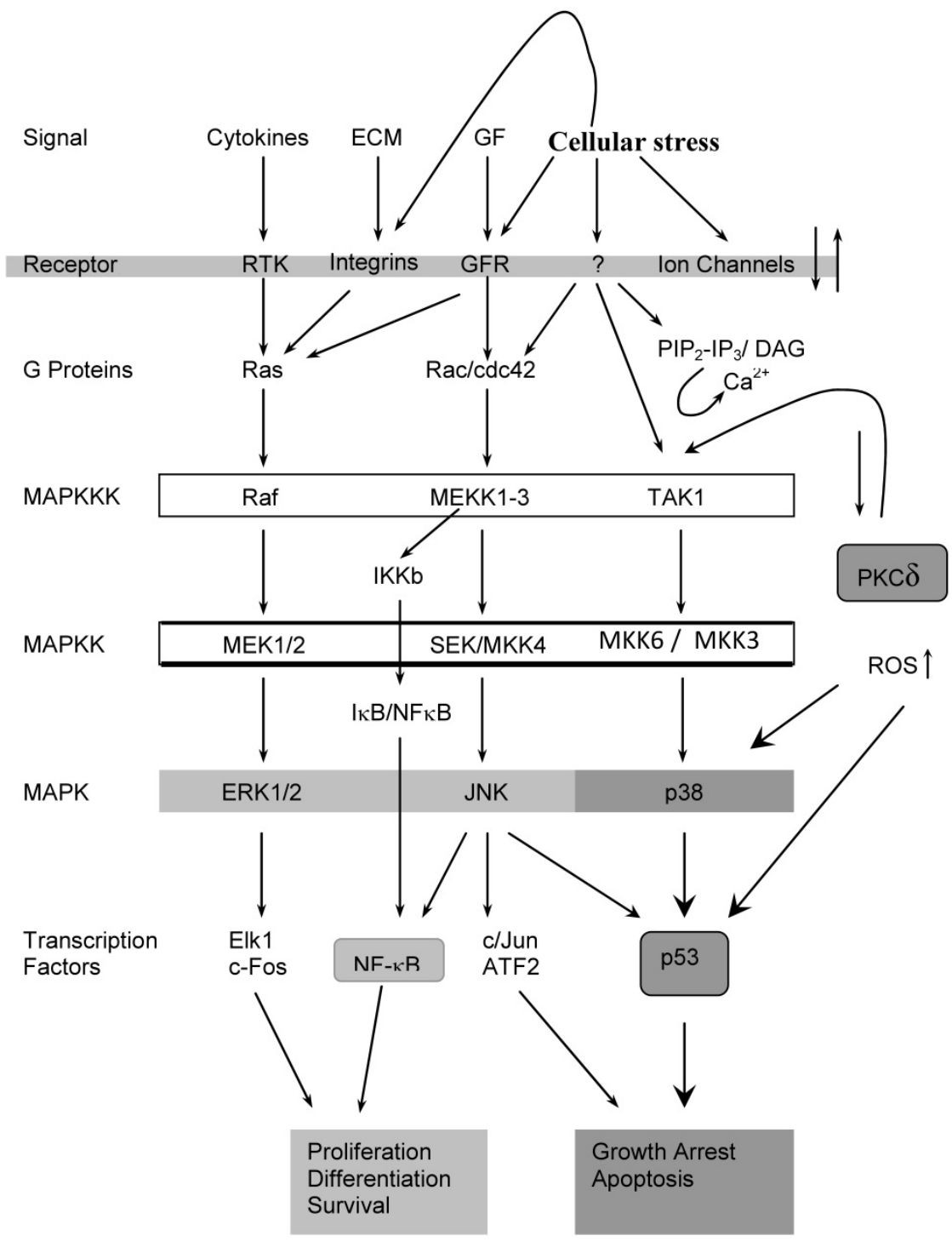

Figure 4. MAPK activation in stress-induced signalling in the heart. A variety of stress signals can activate the MAPKs directly or indirectly. MAPKs comprise a family of tyrosine/threonine kinases. Receptor activation initiates a cascade of phosphorylation events involving sequential activation of $\mathrm{G}$ proteins, MAPK kinase kinase (MAPKKK), MAPK kinase (MAPKK) and finally MAPK. Activated MAPK, in turn, is responsible for the phosphorylation and activation of various other regulatory proteins and transcription factors, which induce the expression of genes involved in the regulation of cell proliferation and apoptosis. ERK kinases mediate cell survival and proliferation, whereas JNK and p38 induce growth arrest and apoptosis (modified from Wernig and Xu, 2002). 
Studies using chemical inhibitors have led to the conclusion that activation of the p38MAPK promotes cardiac myocyte death during extended periods of ischemia [69-71]. In a cultured neonatal rat cardiac myocyte model, inhibition of p38-MAPK protects against ischaemic injury by decreasing LDH release $[69,70]$. In addition, Barancik and co-workers (2000) reported that a specific inhibitor of p38-MAPK, SB203580, protected pig myocardium against ischaemic injury in an in vivo model by reducing infarct size [71]. Several studies indicated that p38-MAPK plays a pivotal role in promoting myocardial apoptosis [69,72-73]. Ma and co-workers (1999) demonstrated that in isolated perfused rabbit hearts, ischemia alone caused a moderate but transient increase in p38-MAPK activity [72]. Ten minutes' reperfusion further activated $\mathrm{p} 38$-MAPK, which remained elevated throughout reperfusion (20 minutes). Administration of SB203580 before ischemia and during reperfusion completely inhibited p38 MAPK activation and exerted significant cardioprotective effects, characterized by decreased myocardial apoptosis and improved post-ischaemic function, as well as attenuated myocardial necrotic injury. In contrast, administering SB203580 10 minutes after reperfusion (a time point when maximal MAPK activation had already been achieved), failed to convey significant cardioprotection. Mackay and Mochly-Rosen (1999) indicated that in neonatal rat cardiac myocytes, two distinct phases of p38 activation were observed during ischemia: the first phase began within 10 minutes and lasted less than 1 hour, and the second began after 2 hours and lasted throughout the ischaemic period [69]. They demonstrated that SB203580 also protected cardiac myocytes against ischemia by reducing activation of caspase-3, a key event in apoptosis. However, the protective effect was seen even when the inhibitor was present during only the second, sustained phase of p38 MAPK activation. Subsequent studies by Yue and co-workers (2000), exposing rat neonatal cardiomyocytes to ischemia showed a rapid and transient activation of p38-MAPK and JNK [73]. On reoxygenation, further activation of SAPKs was noted. With pretreatment of the cells with SB203580 apoptotic cells were reduced, suggesting p38 MAPK activation mediates apoptosis in rat cardiac myocytes subjected to ischemia/reoxygenation. In addition, Yue and co-workers (2000) also showed that SB203580 improved cardiac contractile function in rat isolated ischaemic hearts. Inhibition of p38 MAPK activation, therefore, correlated with cardioprotection against ischemia/reperfusion injury in cardiac myocytes as well as in isolated hearts [73].

Zechner and co-workers (1998) also reported that overexpression of MKK6 (Figure 4), an upstream activator of p38 MAPK, resulted in protection of cardiac myocytes from apoptosis induced either by anisomycin or MEKK1, an upstream activator of the JNK pathway [74]. In addition, expression of MKK6 elicited a hyperthrophic response, which was enhanced by co-infection of p38 $\beta$ [75]. Therefore, a distinct isoform of p38 MAPK, p38 $\beta$, may participate in mediating cell survival. In contrast, over expression of MKK3 in mouse cardiomyocytes led to apoptosis, which was increased by co-infection of p38 $\alpha$ [75]. Therefore differential activation of p38-MAPK isoforms may exert opposing effects: p38 $\alpha$ is implicated in cell death, while $\mathrm{p} 38 \beta$ may mediate myocardial survival.

To determine whether p38 MAPK activation was isoform selective, rat neonatal cardiomyocytes were infected with adenovirus encoding wild-type p38 $\alpha$ or p38 $\beta$ [70]. They 
showed that transfected p38 $\alpha$ and p38 $\beta$ were differentially activated during sustained ischemia, with p38 $\alpha$ remaining activated but p38 $\beta$ deactivated. Furthermore, cells expressing a dominant negative $\mathrm{p} 38 \alpha$, which prevented ischemia-induced p38 MAPK activation, were resistant to sustained ischaemic injury. Therefore, activation of p38 $\alpha$ MAPK isoform is detrimental during ischemia.

\section{MAPK inactivation by phosphatases}

Dephosphorylation of either the threonine or tyrosine residue within the MAPK activation loop TxY motif alone can result in their enzymatic inactivation. In intact cells, dephosphorylation and inactivation of MAPK occur, within minutes to several hours depending on the cell type and activating stimulus. In endothelial cells, exposure to serum leads to ERK activation that is sustained at high levels for over $2 \mathrm{~h}$. In contrast, different patterns can be observed in the a PC12 cell line where EGF-stimulated ERK activation is transient, with inactivation initiated within $5 \mathrm{~min}$ and nearly complete within 15-30 min, whereas this MAPK displays prolonged activation for several hours on stimulation with NGF [76]. It is believed that different patterns of ERK activation elicited by EGF and NGF underlie their differential effects to drive either cellular proliferation of differentiation, respectively [77]. Using PC12 cells as a model system to identify key phosphatases suppressing ERK activation, biochemical studies revealed that early rapid inactivation of these MAPKs reflects, in part, threonine dephosphorylation by the serine/threonine protein phosphatase PP2A [76].

In addition to threonine dephosphorylation, these studies also indicated that tyrosinespecific protein phosphatases (PTPs) also contribute to ERK inactivation [76]. Currently, 50 or more PTPs have been characterized [78-80], and although the PC12 cell PTPs were not identified molecularly [76], recent studies in other cell types have identified a possible role for three related PTP gene family members [81-84]. Notwithstanding the importance of these early reports on PP2A and tyrosine-specific PTPs inactivating ERKs, little is known about their general importance in terminating MAPK signaling, of the molecular mechanisms that may control phosphatase catalytic activity, or of their specificity for inactivating different MAPK isoforms.

In contrast to these protein phosphatase classes, there has been significant and rapid progress in our understanding of the role played by a subclass of PTP that possess activity for dephosphorylating both phosphotyrosine and phosphothreonine residues, known as the dual specificity phosphatases (DSPs).

The first mammalian DSP was identified as the mouse immediate early gene 3CH134 or its human orthologue CL100, which is induced rapidly after exposure to growth factors, heat shock, or oxidative stress [85-87]. Recombinant CL100/3CH134 was shown to dephosphorylate threonine and tyrosine residues of ERK, which was paralleled by its inactivation. These studies showed that CL100/3CH134 was specific for dephosphorylation of ERK when compared to a number of other unrelated phosphoproteins [76]. A correlation between $3 \mathrm{CH} 134$ levels and ERK inactivation was also found in mammalian cells, leading to its renaming as MAPK phosphatase-1 (MKP-1) [88]. Despite this important early work, the 
relevance of MKP-1 in ERK inactivation remains to be elucidated. Firstly, ERK activity is apparently normal after deletion of the MKP-1 gene in mice [89]. Secondly, it has also become evident that MKP-1 is at least as effective in inactivating JNK and p38 when compared to the ERKs [90-91]. Thirdly, newly identified members of the DSP gene family appear highly selective for ERK and may represent the true physiological regulators of this MAPK isoform. Since the initial cloning of MKP-1, eight additional mammalian DSP gene family members have been identified and characterized, which include MKP-2, MKP-3, MKP-4, MKP-5, MKP-X, PAC1, M3/6 and B59. These DSPs all appear to be effective in mediating inactivation of MAPKs.

The following model for MAPK inactivation by DSP is suggested (Figure 5). Stimulation by growth factors, cytokines, cellular stresses or some active oncogenes leads to rapid transcription of one or a subset of DSP genes. Increased DSP transcription may reflect activation of specific MAPK, although alternative pathways are not excluded. After translation of the DSP mRNA into protein, the catalytically inactive DSP translocates to a specific subcellular compartment within either the nucleus or the cytosol. Upon encountering its target MAPK, the DSP binds tightly through its amino terminus, which in turn triggers activation of the phosphatase catalytic domain. If the bound MAPK is already activated, then this will result in its rapid inactivation. Conversely, if the MAPK is not

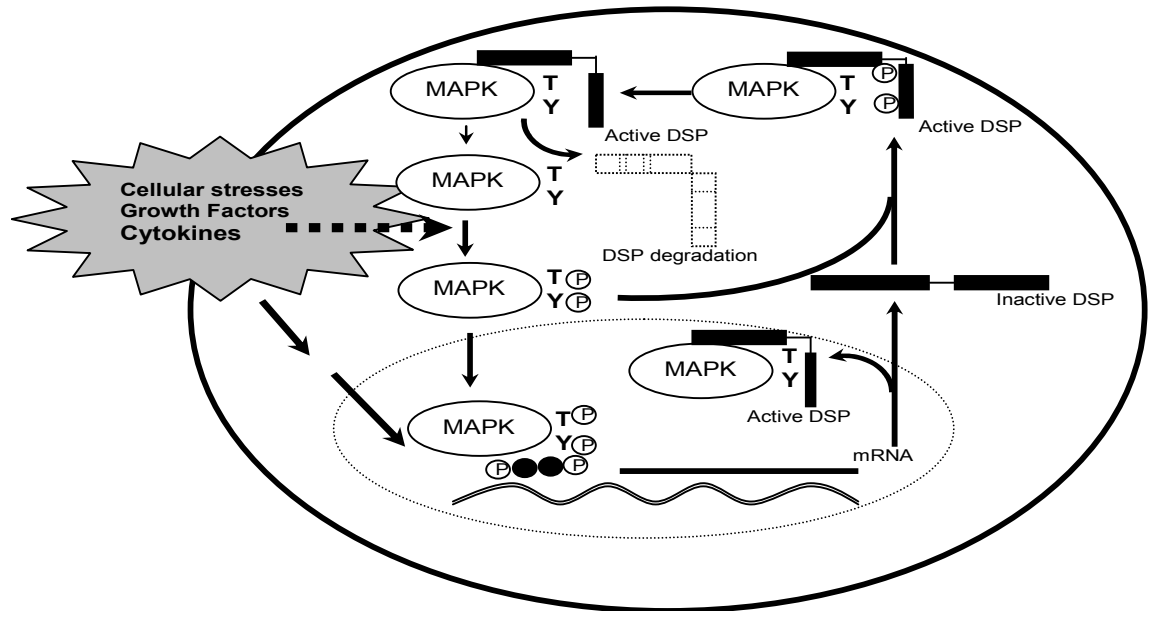

Figure 5. Cell exposure to growth factors, cytokines and cell stresses leads to induction of a subset of DSP genes. Increased expression is likely to reflect activation of transcription factors (black circles) via both MAPK-dependent and independent pathways. Newly synthesized DSPs translocate to specific subcellular compartments as dictated by anchorage and/or localization motifs not yet identified. Specific binding to target MAPKs through regions within the DSP amino terminus then triggers activation of the phosphatase catalytic domain. Bound MAPKs are in turn inactivated by dephosphorylation on threonine and tyrosine residues localized within the "activation loop" motif of TxY. Inactive MAPKs then dissociate, leaving the DSP free to bind and inactivate another MAPK molecule. In the absence of continued DSP gene transcription and protein synthesis, rapid degradation may limit their duration of activity in cells (Camps et al., 1999). 
active, then its tight interaction with an active DSP is expected to block any possibility of kinase activation by a subsequent stimulus. MAPKs that fail to bind the DSP within its amino terminus remain active or susceptible to activation after extracellular stimulation. Depending on their cellular localization, these regulatory effects allow for selected inhibition of MAPK activities in specific subcellular compartments. Some DSPs have been shown to possess short half-lives [76], suggesting that in the absence of continued gene transcription and protein synthesis, their rapid turnover limits their duration of action in cells. Overall, tight control of DSP gene induction, combined with their differential binding and catalytic activation by a specific repertoire of MAPKs, provides a sophisticated mechanism for rapid targeted inactivation of selected MAPK activities.

\section{MAP kinase phosphatase-1 (MKP-1): A mediator of the beneficial effects of glucocorticoids during ischemia/reperfusion-induced stress in the heart}

It is noteworthy that many genes that are positively regulated at post-transcriptional level by $\mathrm{p} 38-\mathrm{MAPK}$, are negatively regulated at the same level by glucocorticoids. Lasa and coworkers investigated the effects of GCs on the p38-MAPK pathway and have shown that dexamethasone destabilized cyclooxygenase-2 (COX-2) mRNA by inhibiting the function, but not the expression of p38-MAPK [92]. The inhibition of p38-MAPK was then shown to be mediated by MKP-1. We and others have also demonstrated that dexamethasone induces the expression of MKP-1 which potently inactivated p38-MAPK [93-94].

$\mathrm{Wu}$ and Bennet (2005) demonstrated that MKP-1 promotes cell survival in fibroblasts through the attenuation of stress responsive MAPK-mediated apoptosis [95]. Upregulation of MKP-1 has also been shown to be associated with cardioprotection by long-chain polyunsaturated fatty acids [93]. It has also been reported that transgenic mice overexpressing MKP-1 were protected, whereas knock-out mice show greater injury after ischemia/reperfusion [96]. The exact mechanism of the beneficial effects of glucocorticoids on the heart during ischemia/reperfusion-induced stress still remain to be established. In view of the significant contribution of apoptosis, necrosis and autophagy during ischemia/reperfusion-induced stress, it is expected that GC-induced cardioprotection to be associated with reduced apoptosis and necrosis. Indeed, Fan and co-workers have demonstrated that dexamethasone, administered intraperitoneally or added directly to the perfusate, significantly improved post-ischemic functional recovery and reduced infarct size compared to untreated controls [94]. These were associated with associated with upregulation of MKP-1 protein expression [94]. Furthermore, it was also shown by us that upregulation of MKP-1 during simulated ischemia/reperfusion is associated with an attenuation of apoptosis in neonatal cardiomyocytes [93].

\section{Conclusions}

It has been suggested that upregulation of MKP-1 during ischemia/reperfusion-induced stress attenuates myocardial injury $[93,94]$. MKP-1, found predominantly in the nucleus, is a 
dual specific phosphatase which dephosphorylates phosphotyrosine and phosphothreonine-containing protein kinases such as the MAPKs. MAPKs are known to be involved in intracellular signalling pathways that regulate gene expression in response to a variety of extracellular signals. MAPKs are activated during ischemia/reperfusion-induced stress in the heart. It was also demonstrated that glucocorticoids act via MKP-1 induction and subsequent p38-MAPK inhibition to induce cardioprotection during ischemia/reperfusion-induced stress [94].

MAPKs have been found to be involved in autophagic, apoptotic and necrotic cell death during stress responses of the heart [97-102]. Autophagy is foremost a survival mechanism which is activated in cells subjected to nutrient or growth factor deprivation. However, when the cellular stress continues, cell death may occur via autophagy, or becomes associated with features of apoptotic or necrotic cell death [103]. Apoptosis is essential for removal of specifically targeted cells, through the process of apoptotic body formation and phagocytosis [104]. Necrosis is a pathological cellular response requiring no ATP. Necrotic cells are morphologically characterized by disrupted membranes, cytoplasm and mitochondrial swelling, disintegration of organelles and complete cell lysis [105]. Cell death following ischemia/reperfusion-induced stress is thought to manifest in morphological features indicative for all three, apoptotic, necrotic and autophagic cell death [106].

MKP-1 has been shown to be involved in the regulation of apoptosis [107] and it was also very recently demonstrated that MKP-1 may lead to autophagy induction in cancer cells [108]. We have recently demonstrated that inhibition of MKP-1 and subsequent increased p38-MAPK phosphorylation during ischemia/reperfusion-induced stress is associated with attenuated autophagy and increased apoptosis and necrosis in the heart (unpublished data). We thus propose the following mechanism of GC-induced protection in the heart: During ischemia/reperfusion-induced stress in the heart, p38 MAPK is activated, GCs sustain/upregulate autophagy via an increase in MKP-1 and subsequent dephosphorylation of p38 MAPK which ultimately protects the heart from apoptosis and necrosis, driven by the effects of autophagy on the metabolic balance sheet of the heart.

\section{Author details}

Anna-Mart Engelbrecht* and Benjamin Loos

Dept of Physiological Sciences, Stellenbosch University, Stellenbosch, South Africa

\section{References}

[1] Dallman MF, Strack AM, Akana SF, Bradbury MJ, Hanson ES, Scribner KA (1993) Feast and famine: critical role of glucocorticoids with insulin in daily energy flow. Fron Neuroendocrinol 14: 303-347.

\footnotetext{
${ }^{*}$ Corresponding Author
} 
[2] Hammond GL, Smith CL, Paterson NAM, Sibbald WJ (1990) A role for corticosteroidglobulin in delivery of cortisol to activated neutrophils. J Clin Endocrinol Metab 71: 3439.

[3] Buttgereit F, Scheffod A (2002) Rapid glucocorticoid effects on immune cells. Steroids 6: 529-534.

[4] Stellato C (2004) Port-transcriptional and nongenomic effects of glucocorticoids. Proc Am Thorac Soc 1: 255-263.

[5] Limbourg FP, Liao JK (2003) Nontranscriptional actions of the glucocorticoid receptor. J Mol Med 81: 168-174.

[6] Wehling M (1997) Specific, nongenomic actions of steroid hormones. Annu Rev Physiol 59: 365-393.

[7] Ito K, Chung KF, Adcock IM (2006) Update on glucocorticoid action and resistance. J Allergy Clin Immunol 117: 522-543.

[8] Clark AR, Dean JL, Saklatvala J (2003) Post-transcriptional regulation of gene expression by mitogen-activated protein kinase p38. FEBS Letters 546: 37-44.

[9] Sapolsky RM, Romero LM, Munck AU (2000) How do glucocorticoids influence stress responses? Integrating permissive, suppressive, stimulatory, and preparative actions. Endocr Rev 21: 55-89.

[10] Libby P, Maroko PR, Bloor CM, Sobel BE, Braunwald E (1973) Reduction of experimental myocardial infarct size by corticosteroid administration. J Clin Invest 52: 599-607.

[11] Valen G, et al (2000) Glucocorticoid pre-treatment protects cardiac function and induces cardiac heat shock protein 72. Am J Physiol Heart Circ Physiol 279: H836-H843.

[12] Varga E, et al (2004) Inhibition of ischemia/reperfusion-induced damage by dexamethasone in isolated working rat hearts: the role of cytochrome c release. Life Sci 75: 2411-2423.

[13] Skyschally A, et al (2004) Glucocorticoid treatment prevents progressive myocardial dysfunction resulting from experimental coronary microembolization. Circulation 109: 2337-2342.

[14] Giugliano GR, Giugliano RP, Gibson CM, Kuntz RE (2003) Meta-analysis of corticosteroid treatment in acute myocardial infarction. Am J Cardiol 91: 1055-1059.

[15] Dennis SC, Gevers W \& Opie LH (1991) Protons in ischemia: where do they come from: where do they go to? J Mol Cell Cardiol 23: 1077-1086.

[16] Zong WX \& Thompson SB (2006) Necrotic death as a cell fate. Genes Dev 20: 1-15.

[17] Murry CE, Jennings RB \& Reimer KA (1986) Preconditioning with ischemia: a delay of lethal cell injury in ischemic myocardium. Circulation 74: 1124-1136.

[18] Nishida K, Kyoi S, Yamaguchi O, Sadoshima J \& Otsu K (2009) The role of autophagy in the heart. Cell Death Differ 16: 31-38.

[19] Olivetti G, Abbi R, Quaini F, Kajstura J, Cheng W, Nitahara JA, Quaini E, Di Loreto C, Beltrami CA, Krajewski S, Reed JC \& Anversa P (1997) Apoptosis in the failing human heart. N Engl J Med 336: 1131-1141.

[20] De Duve C \& Wattiaux R (1966) Functions of Lysosomes. Ann Rev Physiol 28: 435-92. 
[21] Mizushima N, Levine B, Cuervo AM, Klionsky DJ (2008) Autophagy fights disease through cellular self-digestion. Nature 451(7182):1069-75.

[22] Baba M, Takeshige G, Baba N and Ohsumi Y (1994) Ultrastructural analysis of the autophagic process in yeast: Detection of autophagosomes and their characterization. J Cell Biol 6:903-13.

[23] Dice FJ (2007) Chaperone-Mediated Autophagy. Autophagy 4:295-9.

[24] He C, Klionskly DJ (2009) Regulation mechanisms and signaling pathways in autophagy. Annu Rev Genet 43:67-93.

[25] Cuervo AM (2010) The plasma membrane brings autophagosomes to life. Nat.Cell Biol12: 735-737.

[26] Cuervo AM (2004) Autophagy: Many paths to the same end. Mol Cell Biochem 263:5572.

[27] Yorimitsu T and Klionsky DJ (2005) Autophagy: molecular machinery for self-eating. Cell Death Differ 12:1542-1552.

[28] Fass E, Amar N and Elazar Z (2007). Identification of essential residues for the cterminal cleavage of mammalian LC3. Autophagy 3:1:48-50.

[29] Scherz-Shouval R, Shvets E, Elazar Z (2007) Oxidation as a post-translational modification that regulates autophagy. Autophagy 4:371-3.

[30] Sybers HD, Ingwall J, DeLuca M (1978) Autophagy in cardiac myocytes. Recent Adv Stud Cardiac Struct Metab 12:453-463.

[31] Hamacher-Brady A, Brady NR, Gottlieb RA (2006) Enhancing macroautophagy protects against ischemia/reperfusion injury in cardiac myocytes. J Biol Chem 281: 29776-29787.

[32] Loos B, Engelbrecht AM (2009). Cell death: a dynamic response concept. Autophagy 5(5):1-14.

[33] Loos, B; Genade, S.; Ellis, B.; Lochner, A.; Engelbrecht, A. M (2011). At the core of survival: Autophagy delays the onset of both apoptotic and necrotic cell death in a model of ischemic cell injury. Exp Cell Res 317: 1437-1453.

[34] Matsui Y, Takagi H, Qu X, Abdellativ M, Sakoda H, Asano T (2007) Distinct roles of autophagy in the heart during ischemia and reperfusion: roles of AMP-activated protein kinase and beclin 1 in mediating autophagy. Circ Res100: 914-922.

[35] Dosenko VE, Nagibin VS, Tumanovska LV and Moibenko AA (2006) Protective effect of autophagy in anoxia-reoxygenation of isolated cardiomyocytes? Autophagy 2(4): 305306.

[36] Yue Z, Jin S, Yang C, Levine AG, Heintz N (2003). Beclin 1, an autophagy gene essential for early embryonic development, is a haploinsufficient tumor suppressor. Proc Natl Acad Sci USA; 100: 15077-15082.

[37] Nakai A, Yamaguchi O, Takeda T, Higuchi Y, Hikoso S, Taniike M (2007) The role of autophagy in cardiomyocytes in the basal state and in response to hemodynamic stress. Nat Med 13: 619-624.

[38] Gustafsson $\AA B$, Gottlieb RA (2009). Autophagy in ischemic heart disease. Circ Res 104:150-158.

[39] Lam A, Lopaschuk GD (2007) Anti-anginal effects of partial fatty acid oxidation inhibitors. Curr Opin Pharmacol 7:179-185. 
[40] Altarejos JY, Taniguchi M, Clanacham AS, Lopaschuk GD (2005). Myocardial ischemia differentially regulates LKB1 and an alternate 5'-AMP-activated protein kinase kinase. J Biol Chem 280:183-190.

[41] Jafri MS, Dudycha SJ, O'Rourke B (2001). Cellular energy metabolism: models of cellular respiration. Annu Rev Biomed Eng 3:57-81.

[42] Takagi H, Matsui Y, Sadoshima J (2007). The role of autophagy in mediating cell survival and death during ischemia and reperfusion in the heart. Antioxid Redox Signal 9:1373-1381.

[43] Grover GJ, Atwal KS, Sleph PG, Wang FL, Monshizadegan H, Monticello T (2004) Excessive ATP hydrolysis in ischemic myocardium by mitochondrial F1F0 ATPase: effect of selective pharmacological inhibition of mitochondrial ATPase hydrolase activity. Am J Physiol Heart Circ Physiol 287: 1747-1755.

[44] Kunapuli S, Rosanio S, Schwarz ER (2006) "How do cardiomyocytes die?" Apoptosis and autophagic cell death in cardiac myocytes. J Card Fail 12:381-391.

[45] Leist M, Single B, Castoldi AF, Kuhnle S, Nicotera P (1997) Intracellular adenosine triphosphase (ATP) concentration: A switch in the decision between apoptosis and necrosis. J Exp Med 185: 1481-6.

[46] Tatsumi T, Shiraishi J, Keira N, Akashi K, Mano A, Yamanaka S (2003) Intracellular ATP is required for mitochondrial apoptotic pathways in isolated hypoxic rat cardiac myocytes. Cardiovasc Res 59: 428-40.

[47] Singh R and Cuervo AM (2011). Autophagy in the cellular energetic balance. Cell Metabolism 13: 495-504

[48] Mizushima N and Klionsky DJ (2007) Protein turnover via autophagy: implications for metabolism. Annu Rev Nutr 27:19-40.

[49] Singh R , Kaushik S, Wang Y, Xiang Y, Novak I, Komatsu M, Tanaka K, Cuervo AM and Czaja MJ (2009) Autophagy regulates lipid metabolism. Nature 458: 1131-1135.

[50] Nemchenko A, Chiong M, Turer A, Lavandero S, Hill JA (2011). Autophagy as therapeutic target in cardiovascular disease. JMCC 51: 584-593.

[51] Juhász G, Csikós G, Sinka R, Erdélyi M, Sass M (2003) The Drosophila homolog of Aut1 is essential for autophagy and development. FEBS Lett 543: 154-8.

[52] Levine B and Klionsky DJ (2004). Development by self-digestion: molecular mechanisms and biological functions of autophagy. Developmental Cell 6: 463-477.

[53] Martin DN, Balgley B, Dutta S, Chen J, Rudnick P, Cranford J, Kantartzis S, DeVoe DL, Lee C, Baehrecke EH (2007) Proteomic analysis of steroid-triggered autophagic programmed cell death during Drosophila development. Cell Death Differ 14: 916-23.

[54] Kuma A, Hatano M, Matsui M, Yamamoto A, Nakaya H, Yoshimori T, Ohsumi Y, Tokuhisa T, Mizushima N (2004) The role of autophagy during the early neonatal starvation period. Nature 432: 1032-6.

[55] Yue Z, Jin S, Yang C, Levine AG, Heintz N (2003). Beclin 1, an autophagy gene essential for early embryonic development, is a haploinsufficient tumor suppressor. Proc Natl Acad Sci USA 100: 15077-15082.

[56] Yu L, Alva A, Su H, Dutt P, Freundt E, Welsh S (2004) Regulation of an ATG7-beclin 1 program of autophagic cell death by caspase-8. Science 304: 1500-1502. 
[57] Shimizu S, Kanaseki T, Mizushima N, Mizuta T, Arakawa-Kobayashi S, Thompson CB and Tsujimoto $\mathrm{Y}$ (2004) Role of Bcl-2 family proteins in a non-apoptotic programmed cell death dependent on autophagy genes. Nature Cell Biology 6: 1221 - 1228.

[58] Kinch G, Hoffman KL, Rodrigues EM, Zee MC, Weeks JC (2003) Steroid-triggered programmed cell death of a motorneuron is autophagic and involves structural changes in mitochondria. J Comp Neurol 17: 384-403.

[59] Khamzina L, Veilleux A, Bergeron S, Marette A (2005). Increased activation of the mammalian target of rapamycin pathway in liver and skeletal muscle of obese rats: possible involvement in obesity-linked insulin resistance. Endocrinology 146:1473-1481.

[60] Hadoke PWF, Iqbal J, Walker BR (2009). Therapeutic manipulation of glucocorticoid metabolism in cardiovascular disease. Brit J Pharmacol 156: 689-712.

[61] Laane E, Tamm KP, Buentke E, Ito K, Khahariza P, Oscarsson J, Corcoran M, Bkörklund AC, Hultenby K, Lundin J, Heyman M, Söderhäll S, Mazur J, Porwit A, Pandolfi PP, Zhivotovsky B, Panaretakis T and Grandér D (2009). Cell death induced by dexamethasone in lymphoid leukemia is mediated through initiation of autophagy. Cell Death Differ 16: 1018-1029.

[62] Cadwell K, Liu JY, Brown SL, Miyoshi H, Loh J, Lennerz JK, Kishi C, Kc W, Carrero JA, Hunt S, Stone CD, Brunt EM, Xavier RJ, Sleckman BP, Li E, Mizushima N, Stappenbeck TS, Virgin HW (2008) A key role for autophagy and the autophagy gene Atg16 in mouse and human intestinal Paneth cells. Nature 13(456): 259-63.

[63] Deretic V (2009) Links between autophagy, innate immunity, inflammation and Chron's disease. Dig Dis 27:246-251.

[64] Begum N, Ragolia L, Rienzie J, McCarthy M \& Duddy N (1989) Regulation of mitogenactivated protein kinase phosphatase-1 induction by insulin in vascular smooth muscle cells. Evaluation of the role of the nitric oxide signalling pathway and potential defects in hypertension. J Biol Chem 273: 25164-25170.

[65] Cowan KJ \& Storey (2003) Mitogen-activated protein kinases: new signalling pathways functioning in cellular responses to environmental stress. J Exp Biol 206: 1107-1115.

[66] Marczin N, El-Habashi N, Hoare GS, Bundy RE \& Yacoub M (2003) Antioxidants in myocardial ischemia-reperfusion injury: therapeutic potential and basic mechanisms. Arch Biochem Biophys 420: 222-236.

[67] Junttila MR, Li SP, Wetermarck J (2008) Phosphatase-mediated crosstalk between MAPK signalling pathways in the regulation of cell survival. Faseb J 22: 954-965.

[68] Weston CR \& Davis RJ (2007) The JNK signal transduction pathway. Curr Opin Cell Biol 19: 142-149.

[69] Mackay K, Mochly-Rosen D (2001) Arachidonic acid protects neonatal rat cardiac myocytes form ischaemic injury through $\varepsilon$ protein kinase C. Cardiovasc Res 50: 65-74.

[70] Saurin AT, Martin JC, Heads RJ, Foley C, Mockridge JW, Wright MJ, Wang Y, Marber S (2000) The role of differential activation of p38 mitogen activated protein kinases in preconditioned ventricular myocytes. Faseb J 14: 2237-2246.

[71] Barancik M, Htun P, Strohm C, Killian S, Schaper W (2000) Inhibition of the cardiac p38MAPK pathway by SB20350 delays ischemic cell death. J Cardiovasc Pharmacol 35: 474483. 
[72] Ma XL, Kumar S, Gao F, Louden CS, Lopez BL, Christopher TA, Wang C, Lee JC, Feuerstein GZ, Yue TL (1999) Inhibition of p38 mitogen-activated protein kinase decreases cardiomyocyte apoptosis and improves cardiac function after myocardial ischemia and reperfusion. Circulation 99: 1685-1691.

[73] Yue TL, Wang C, Gu J-L, Ma XL, Kumar S, Lee JC, Feuerstein GZ, Thomas H, Maleeff B, Ohlstein EH (2000) Inhibition of extracellular signal-regulated kinase enhances ischemia/reoxygenation-induced apoptosis in cultured cardiac myocytes and exaggerates reperfusion injury in isolated perfused hearts. Circ Res 86: 692-699.

[74] Zechner D, Craig R, Hanford HS, McDonough PM, Sabbadini RA, Glembotski CC (1998) MKK6 activates myocardial cell NF-kappaB and inhibits apoptosis in a p38 mitogen-activated protein kinase-dependent manner. J Biol Chem 273: 8232-8239.

[75] Wang X, Martindale JL, Liu Y, Holbrook NJ (1998) The cellular response to oxidative stress: influences of mitogen-activated protein kinase signalling pathways on cell survival. Biochem J 333: 291-300.

[76] Alessi DR, Gomez N, Moorhead G, Lewis T, Keyse SM, Cohen P (1995) Inactivation of p42 MAPK kinase by protein phosphatase $2 \mathrm{~A}$ and a protein tyrosine phosphatase, but not CL 100, in various cell lines. Curr Biol 5: 283-295.

[77] Marshall CJ (1995) Specificity of receptor tyrosine kinase signalling: transient versus sustained extracellular signal-regulated kinase activation. Cell 80: 179-185.

[78] Neel BG, Tonks NK (1997) Protein tyrosine phosphatases in signal transduction. Curr Opin Cell Biol 9: 193-204.

[79] Hooft van Huijsduijnen R (1998) Protein tyrosine phosphatases: counting trees in the forest. Gene 225: 1-8.

[80] Denu JM, Dixon JE (1998) Protein tyrosine phosphatases: mechanisms of catalysis and regulation. Curr Opin Chem Biol 2: 663-641.

[81] Wurgler-Murphy SM, Maeda T, Witten EA, Saito H (1997) Regulation of the Saccharomyces cerevisiae HOG1 mitogen activated protein kinase by PTP2 and PTP3 protein tyrosine phosphatases. Mol Cell Biol 15: 1289-1297.

[82] Shiozaki K, Russel P (1995) Cell-cycle control linked to extracellular environment by MAP kinase pathway in fission yeast. Nature 378: 739-743.

[83] Millar JB, Buck V, Wilkinson MG (1995) Pyp1 and Pyp2 PTPases dephosphorylated an osmosensing MAP kinase controlling cell size at division in fission yeast. Genes Dev 9: 2117-2130.

[84] Zhan X-L, Deschenes RJ, Guan KL (1997) Differential regulation of FUS3 MAP kinase by tyrosine-specific phosphatases PTP2/PTP3 and dual-specificity phosphatase MSG5 in Saccharomyces cervisiae. Genes Dev 11: 1690-1702.

[85] Keyse SM \& Emslie EA (1992) Oxidatives stress and heat shock induce a human gene encoding a protein-tyrosine phosphatase. Nature 359: 644-646.

[86] Charles CH, Sun H, Lau LF, Tonks NK (1993) MKP-1, an immediate early gene product is a dual specificity phosphatase that dephosphorylates MAP kinases in vivo. Cell 75(3): 487-493.

[87] Noguchi T, Metz R, Chen L, Mattei MG, Carasco D, Bravo R (1993) Structure, mapping and expression of erp, a growth factor-inducible gene encoding a nontransmembrane 
protein tyrosine phosphatase, and effect of ERP on cell growth. Mol Cell Biol 13: 51955205.

[88] Sun H, Charles CH, Lau LF, Tonks NK (1993) MKP-1 (3Ch134), an immediate early gene product, is a dual specificity phosphatase that dephosphorylates MAP kinase in vivo. Cell 75: 487-493.

[89] Dorfman K, Carrasco D, Gruda M, Ryan C, Lira SA, Bravo R (1996) Disruption of the erp/mkp-1 gene does not affect mouse development: normal MAP kinase activity in ERP/MKP-1-deficient fibroblasts. Oncogene 13: 925-931.

[90] Chu Y, Solski PA, Khosravi-Far R, Der CJ, Kelly K (1996) the mitogen-activated protein kinase phosphatases PAC1, MKP-1 and MKP-2 have unique substrate specificities and reduced activity in vivo towards ERK2 sevenmaker mutation. J Biol Chem 271: 64976501.

[91] Franklin CC, Kraft AS (1997) Conditional expression of the mitogen-activated protein kinase (MAPK) phosphatase MKP-1 preferentially inhibits p38 MAPK and stressactivated protein kinase in U937 cells. J Biol Chem 272: 16917-16923.

[92] Lasa M, Abraham SM, Boucheron C, Saklatvala J, Clark AR (2002) Dexamethasone causes sustained expression of mitogen-activated protein kinase (MAPK) phosphatase 1 and phosphatase-mediated inhibition of MAPK p38. Mol Cel Biol 22: 7802-7811.

[93] Engelbrecht A-M, Engelbrecht P, Genade S, Niesler C, Page C, Smuts M, Lochner A (2005) Long-chain polyunsaturated fatty acids protect the heart against ischemia/reperfusion-induced injury via a MAPK dependent pathway. J Mol Cell Cardiol 39: 940-954.

[94] Fan WJ, Genade S, Genis A, Huisamen B, Lochner A (2009) Dexamethasone-induced cardioprotection: a role for the phosphatase MKP-1? Life Sci 84: 838-846.

[95] Wu JJ \& Bennett AM (2005) Essential role for mitogen-activated protein (MAP) kinase phosphatase-1 in stress-responsive MAPK and cell survival signalling. J Biol Chem 280: 16461-16466.

[96] Kaiser RA, Bueno OF, Lips DJ, Doevendans PA, Jones F, Kimball TF, Molkentin JD (2004) Targeted inhibition of p38 mitogen-activated protein kinase antagonizes cardiac injury and cell death following ischemia-reperfusion in vivo. J Biol Chem 279: 155241530.

[97] Lee TH, Huang Q, Oikemus S, Shank J, Ventura JJ, Cusson N, Vaillancourt RR, Su B, Davis RJ, Kelliher MA (2003) The death domain kinase RIP1 is essential for tumor necrosis factor alpha signalling to p38 mitogen-activated protein kinase. Mol Cell Biol 23: 8377-8385.

[98] Khan TA, Bianchi C, Ruel M, Voisine P, Sellke FW (2004) Mitogen-activate protein kinase pathways and cardiac surgery. J Thorac Cardiovasc Surg 127: 806-811.

[99] Lee TH, Cusson N, Kelliher MA (2004) The kinase activity of Rip1 is not required for tumor necrosis factor alpha-induced IkappaB kinase or p38 MAP kinase activation or for ubiquitination of Rip1 by Traf2. J Biol Chem 279: 33185-33191.

[100] Codongo P \& Meijer AJ (2005) Autophagy and signalling: Their role in cell survival and cell death. Cell Death Differ 12(2): 1509-1518. 
[101] Park KJ, Lee SH, Lee CH, Jang JY, Chung J, Kwon MH, Kim YS (2009) Upregulation of beclin-1 expression and phosphorylation of Bcl-2 and p53 are involved in the JNKmediated autophagic cell death. Biochem Biophys Res Commun 382: 762-729.

[102] Yang LY, Wu KH, Chiu WT, Wang SH \& Shih CM (2009) The cadmium-induced death of mesangial cells results in nephrotoxicity. Autophagy 5: 571-572.

[103] Maiuri MC, Zalckvar E, Kimchi A, Kroemer G (2007) Self-eating and self-killing: crosstalk between autophagy and apoptosis. Nat Rev Mol Cell Biol 8: 741-752.

[104] Peter C, Waibel M, Radu CG, Yang LV, Witte ON, Schulze-Osthoff K, Wesselborg S, Lauber K (2008) Migration to apoptotic "find-me" signals is mediated via the phagocyte receptor G2A. J Biol Chem 283: 5296-5305.

[105] Zong WX \& Thompson CB (2006) Necrotic death as a cell fate. Genes Dev 20: 1-15.

[106] Murphy E \& Steenbergen C (2008) Mechanisms underlying acute protection from cardiac ischemia-reperfusion injury. Physiol Rev 88: 581-609.

[107] Morisco C, Marrone C, Trimarco V, Crispo S, Monti MG, Sadoshima J, Trimarco B (2007) Insulin resistance affects the cytoprotective effect of insulin in cardiomyocytes through an impairment of MAPK phosphatase-1 expression. Cardiovasc Res 76: 453464.

[108] Lu HH, Kao SY, Liu TY, Liu ST, Huang WP, Chang KW, Lin SC (2010) Areca nut extract induced oxidative stress and upregulated hypoxia inducing factor leading to autophagy in oral cancer cells. Autophagy 6: 725-737. 